\title{
Training Collar-sensed Gestures for Canine Communication
}

Joelle Alcaidinho

Giancarlo Valentin

Gregory D. Abowd

Melody Jackson

Georgia Institute of Technology

\{joelle, giancarlo, abowd,

melodymoorejackson\}

$@$ gatech.edu

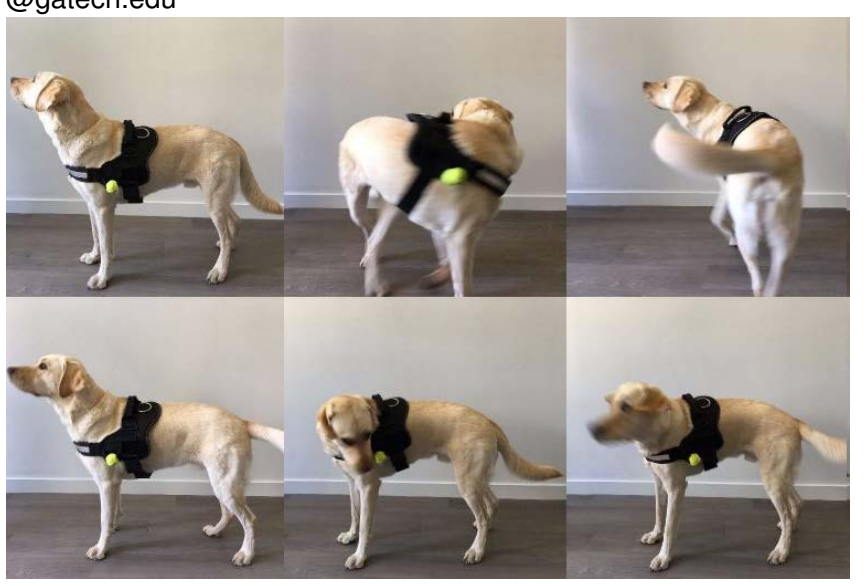

Permission to make digital or hard copies of part or all of this work for personal or classroom use is granted without fee provided that copies are not made or distributed for profit or commercial advantage and that copies bear this notice and the full citation on the first page. Copyrights for third-party components of this work must be honored. For all other uses, contact the owner/author(s).

Copyright held by the owner/author(s).

ACl '16, November 16-17, 2016, Milton Keynes, United Kingdom (1)

http://dx.doi.org/10.1145/2995257.3012020

\begin{abstract}
We describe how we trained two dogs to perform precise gestures to be sensed from an inertial measurement unit worn on a dog's collar as part of a larger research effort. These gestures could then be used to relay specific alerts to humans through a companion smartphone application. For example, a guide dog could use a set of two gestures to alert between obstacles requiring a human to 'wait' or 'go around'.
\end{abstract}

\section{Author Keywords \\ Dog training; Gestures}

\section{ACM Classification Keywords}

H.5.m [Information interfaces and presentation (e.g., $\mathrm{HCl}$ )] Miscellaneous

\section{Introduction}

Information perceived by working dogs often exceeds their ability to communicate it to humans [9]. We have previously proposed using motion gestures sensed from the collar to generate alerts for communication. In this paper, we describe how we selected and trained the first set of gestures.

\section{Gesture selection}

Our first step was to select the gestures the dogs would be trained to perform. We began by considering all potential 


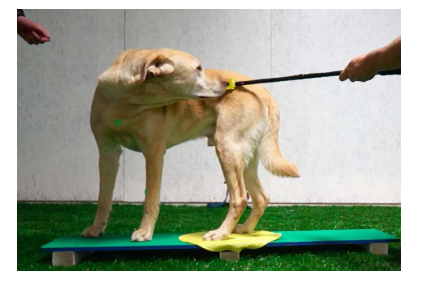

Figure 2: Luring a movement with a target stick collar movements. These were moving the neck in each direction (left, right, up or down). We also considered rotations of the sensor along these dimensions. For example, roll (as in roll over), spin and twirl $\left(360^{\circ}\right.$ clockwise and anticlockwise rotation on yaw axis). Note that a similar movement on the pitch axis would amount to a back flip, which we did not consider. We also discarded roll because even well-trained dogs seemed uncomfortable rolling on the bare floor and it was not clear that dogs would be able to roll on an arbitrary surface.

Vertical gestures (nodding up or down) were difficult to train compared to other gestures because it was harder to elicit these movements. For example, when training a dog, eye contact with the trainer is extremely important. Keeping their heads stationary, so as not to create a false positive indicating the start of a new gesture, until a cue proved to be a challenge. As a last resort, we used a target on the floor during training but this approach led to the impression that the desired action was to touch the floor and wait until rewarded, rather than a nod-like gesture [8]. A full analysis of false positives is presented in Valentin et al. [9].

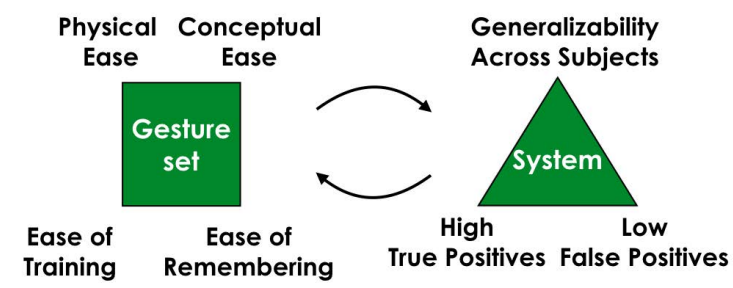

Figure 1: Criteria for ideal gestures. Addressing one aspect tended to affect others.

We summarized the key learnings as seven criteria necessary for suitable gestures (Figure 1) [8]. The gestures that remained were right reach, left reach, spin and twirl.
Reaching left and right were inspired by activation gestures used to trigger wearable interfaces [4]. Spin and twirl are movements taught to some dogs, but not performed regularly enough to be discarded at this stage.

\begin{tabular}{ll}
\hline Candidate Gestures & Description of gestures \\
\hline Spin & Clockwise rotation of $360^{\circ}$ \\
Twirl & Counterclockwise rotation of $360^{\circ}$ \\
Right reach & Reaches to right ribcage and return \\
Left reach & Reaches to left ribcage and return \\
\hline
\end{tabular}

Table 1: The four gestures we examined in this study.

\section{Training considerations}

To avoid training a dog to perform a gesture that would ultimately fail the seven requirements, we first tried to "lure" them into performing each candidate gesture in hopes of prototyping its viability. Luring is a technique by which dogs follow a target object (e.g., treat or toy) to perform an action (Figure 2) [8] . We ultimately realized that the readings from a lured action are more representative of the trainer's luring technique than of the dog's performance, and hence could not be used as a stand-in for the dog performing the gesture on his own. Still, luring was valuable to use in training the dog, but we did not record these instances as examples for the purpose of gesture recognition.

Instead, we had to ensure dogs could learn to offer these gestures after being given a visual or verbal cue. Our first participant had no previous experience with wearable activation interfaces and would not offer actions like "reach left" or "reach right" spontaneously. Our second participant had experience with the gestures but performed them in broad undirected ways when not wearing a wearable activation interface because of the lack of a precise target. 


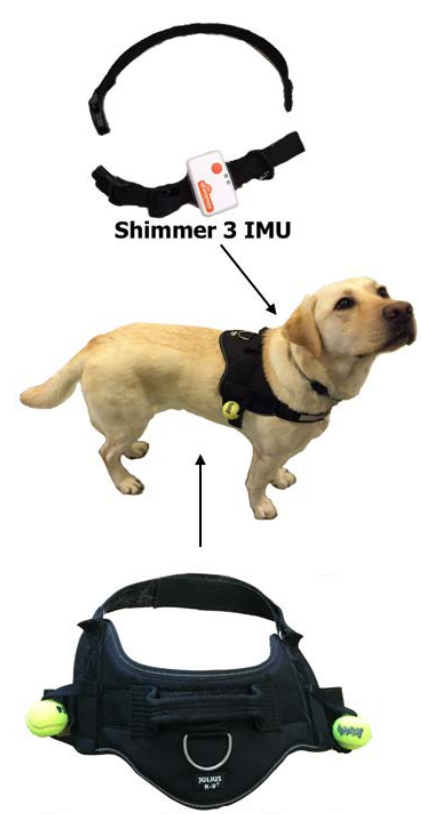

Harness with visual targets

Figure 3: Detail of the instrumented Julius K9 harness to make gestures more precise and the Shimmer3 sensor.
Even though a gestural system did not require a tangible interface it was still necessary to have a visual or tactile target while the dog was learning and until our recognizer could provide feedback upon successful completion.

Based on research bringing humans into the recognition loop, through sonification, we conceived of a system that would use audio feedback throughout a movement [2]. Unfortunately, we found no way of emitting continuous sound without a noticeable delay. Since the sound was also unpleasant to human ears, we speculated it might be aversive to canines. Ultimately, we discovered that using a simple harness-based two-target system was enough to obtain the precision we required (Figure 3 ). Once the gesture was trained, the dog would no longer require the harness.

The harness consisted of two bright colored targets on each side. We built targets out of bright yellow $3.81 \mathrm{~cm}$ diameter balls to make them easier for the dogs to see [5]. Originally, we had used a dark object against a dark background (part of the harness) as a marker, but that was harder to locate as it did not provide enough visual contrast for the dogs [6].

\section{Participants}

For this study, one certified professional dog trainer (CPDTKA), trained two dogs using positive reinforcement. One dog, a two-year-old Retriever (S1) with assistance training, had no experience with wearable interfaces and was trained to perform them exclusively for this experiment. A 7-year old Border Collie (S2), had three years of experience with wearable interfaces.

\section{Training protocol}

When dogs performed (or attempted) a gesture they received a food reward (1 $\mathrm{cm}$ sized treat) [3]. When they could perform the gesture correctly at least $65 \%$ of the times asked, the reinforcement schedule was decreased to only deliver a treat for a correctly performed gesture [1]. Throughout this process the dog also received immediate feedback through the sound of a clicker [7]. For training spin and twirl we relied on luring at the early training stage before transitioning to a subtle hand signal and verbal cue.

Each training session lasted no more than 10 minutes. The average learning time for each gesture varied depending on the dog's prior training experience, but did not exceed more than 15 training sessions per gesture. Gestures were trained both off-leash and on-leash with the dog that had the least experience with wearable devices. The most experienced dog was trained off-leash but showed no observable difference performing the gestures on-leash as long as the leash was long enough to avoid interference.

Due to his experience, we obtained reliable performance from S2 after two practice sessions. We used pronounced hand signals to illustrate the movement for the spin and twirl gestures. Participant S1 was trained intermittently, for two months, until he could perform the gestures from a single verbal cue or minimal hand signals (Figure 4). No food, sensory, or water deprivation was used at any point.

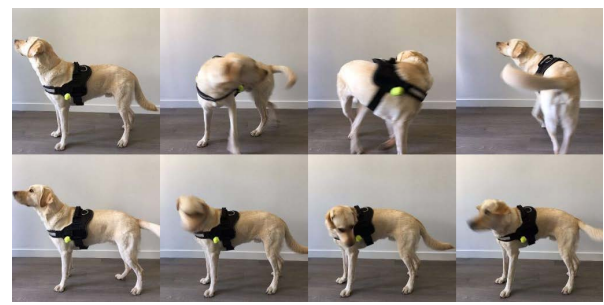

Figure 4: One participant performs a twirl and left reach.

Labeling each example was particularly difficult because 
there was no universally agreed upon definition of where the gesture started and ended. In humans, this requirement is met by enforcing a specific definition on the subject performing the gesture ("you must do it like this, otherwise it will not count."). However, determining the equivalent method for dogs would take much longer.

To optimize for gesture recognition and training time, we decided to train gestures for which we could do the following three items as needed:

- train all of our previous participants (roll would not meet this criteria);

- visually verify gesture completion

- and/ or arbitrarily increase the precision

\section{Conclusion}

The use of the two-target harness was helpful in training the gestures [9]. After this stage, dogs could still perform it without the harness, albeit with less precision. Interestingly, after a three month span, the dogs still remembered the gesture when prompted with a verbal and hand cue, although precision had diminished. In future work, we will examine how these gestures can be associated to specific information that would be present in a real scenario.

\section{Acknowledgements}

This work was supported by the National Science Foundation under Grant IIS-1320690, the GVU Center, the Wearable Computing Center, and Intel Corporation.

\section{REFERENCES}

1. Mariana Bentosela, Gabriela Barrera, Adriana Jakovcevic, Angel M Elgier, and Alba E Mustaca. 2008.

Effect of reinforcement, reinforcer omission and extinction on a communicative response in domestic dogs. In Behavioural Processes, Vol. 78. Elsevier.
2. Gershon Dublon, Laurel S Pardue, Brian Mayton, Noah Swartz, Nicholas Joliat, Patrick Hurst, and Joseph A Paradiso. 2011. DoppelLab: Tools for exploring and harnessing multimodal sensor network data. In SENSORS. IEEE.

3. EF Hiby, NJ Rooney, and JWS Bradshaw. 2004. Dog training methods: their use, effectiveness. In Animal Welfare, Vol. 13. UFAW, 63-70.

4. Melody M Jackson, Giancarlo Valentin, Larry Freil, Lily Burkeen, Clint Zeagler, Scott Gilliland, Barbara Currier, and Thad Starner. 2015. FIDO: wearable communication interfaces for working dogs. In Personal and Ubiquitous Computing, Vol. 19. Springer-Verlag.

5. Paul E Miller and Christopher J Murphy. 1995. Vision in dogs. In Journal-American Veterinary Medical Association, Vol. 207. AVMA, 1623-1634.

6. Jay Neitz, Timothy Geist, and Gerald H Jacobs. 1989. Color vision in the dog. In Visual Neuroscience, Vol. 3. Cambridge Univ Press, 119-125.

7. Karen Pryor. 2009. Reaching the animal mind: clicker training and what it teaches us about all animals. Simon and Schuster, NY.

8. Giancarlo Valentin, Joelle Alcaidinho, Ayanna Howard, Melody M Jackson, and Thad Starner. 2015. Towards a Canine-Human Communication System Based on Head Gestures. In Advances in Computer Entertainment Technology, Vol. 1. ACl, ACM

9. Giancarlo Valentin, Joelle Alcaidinho, Ayanna Howard Melody M Jackson, and Thad Starner. 2016. Creating collar-sensed motion gestures for dog-human communication in service applications. In Proceedings of the 2016 ACM International Symposium on Wearable Computers. ACM, 100-107. 